tools, they are inappropriate for PICUs. We devised an Agreed Limitation of Treatment Form (ALT) for professionals caring for children for whom a decision has been made to withdraw or withhold life-sustaining treatment. We have been using it for 5 years. Recent changes to national guidance have prompted us to review our practice.

Aims To evaluate our Limitation of Treatment process by reviewing our current practice, comparing it to professional guidance and to practice in other PICUs.

Method We compared children for whom an ALT had been completed to children who died without an ALT.

We reviewed each ALT form and the entries in the medical notes.

We obtained the opinions of staff on our PICU.

We reviewed practice in our PICU against the recent changes to professional guidance from the Royal College of Paediatrics and Child Health and the General Medical Council.

We surveyed practice in other units.

Results There were pre-existing severe or life-limiting conditions in $85 \%$ of patients with an ALT compared with $46 \%$ of those who died without an ALT.

13 out of 16 ALT forms were either not fully completed or not consistent with entries in patient notes.

Nursing and medical staff found the form to be useful as a communication tool but requested some changes to the wording and layout of the form.

Our practice is in line with National Guidance.

There is no uniformity among the different PICUs and only a few use forms.

Conclusion We found the Limitation of Treatment forms very useful and consistent with the professional guidance but the quality of documentation needed to be improved. We have made changes to our practice and would suggest that a similar document would be beneficial for all PICUs.

\section{G374 END OF LIFE CARE IN HOSPITAL. SCOPE FOR PAEDIATRIC PALLIATIVE CARE INVOLVEMENT?}

\begin{abstract}
1,2LA Brook, 'K Tewani. 'Specialist Palliative Care Team, Alder Hey Children's Hospital, Liverpool, UK; ${ }^{2}$ International Observatory on Palliative Care, Lancaster University, Lancaster, UK
\end{abstract}

\subsection{6/archdischild-2015-308599.330}

Background End of life care in the setting of choice is important in palliative care. The majority of children die in hospital intensive care where end of life frequently involves withholding/withdrawing life sustaining treatment: many have conditions that may benefit from palliative care. We hypothesised children were dying without specific palliative care support despite a well established paediatric palliative care team and that a proportion of children would be suitable for rapid discharge to the setting of choice for end of life care.

Method Casenotes for inpatient deaths from January to December 2013 were reviewed to identify: children with a life limiting condition, whether the end of life occurred following planned withholding/ withdrawing of life sustaining treatment and if so whether the child was stable enough to be transferred to an alternative care setting for end of life care.

Result 62 children $(31$ male) died. Median age at death was 10 $1 / 2$ months (Q1 6 weeks, Q3 2 years 8 months). Primary diagnoses were cardiac $40 \%$, general paediatrics/neonates $19 \%$, oncology $14 \%$, neurology $11 \%$, congenital malformations $11 \%$. $85 \%$ children had life threatening conditions. 58\% children died following planned withholding/withdrawing of whom 39\% $(\mathrm{N}=$ 14) were stable enough to transfer to an alternative setting for end of life care. Median interval between withholding/withdrawing and death was 30 min but $27 \mathrm{~h}(\mathrm{Q} 1=4 \mathrm{~h}, \mathrm{Q} 3=132 \mathrm{~h})$ for those stable enough to move. All $15 \%(\mathrm{~N}=8)$ children with life limiting conditions known to the palliative care team, but no other children had documented discussion of preferred place of care. Preferred place of care was hospital for 6 and home for 2 of whom both were stable enough to move but not notified to palliative care team until after death.

Conclusion Approximately 22\% children dying in our institution would have been suitable for rapid discharge, living median of $27 \mathrm{~h}$ following transfer to their setting of choice.

\section{G375 CHILDREN WITH LIFE-LIMITING OR COMPLEX CHRONIC CONDITIONS IN PICU; PREVALENCE, WITHIN PICU AND LONG TERM OUTCOMES}

${ }^{1}$ LK Fraser, ${ }^{2} \mathrm{M}$ Van Laar, ${ }^{3} \mathrm{MM}$ Miller, ${ }^{2} \mathrm{RC}$ Parslow. ${ }^{1}$ Department of Health Sciences, University of York, York, UK; ${ }^{2}$ Division of Epidemiology, University of Leeds, Leeds, UK; ${ }^{3}$ Martin House Children's Hospice, Wetherby, UK

\subsection{6/archdischild-2015-308599.331}

Aims This study aimed to review the literature to ascertain what proportion of admission to PICUs are for children with Life Limiting Conditions (LLC) or Complex Chronic Conditions (CCC) and what are their within PICU and post PICU outcomes. Methods: A systematic review of the literature was undertaken with electronic databases, MEDLINE and Embase searched for studies published in English between 1990 until Aug 2014. Hand searching of reference lists was also undertaken.

The titles and abstracts of all the retrieved papers were reviewed for inclusion by two authors. Data was extracted from the full-texts of all the studies which fitted the eligibility criteria. A narrative synthesis of the included studies was undertaken as the heterogeneity of definitions and therefore the included populations and outcomes did not allow for a meta-analysis to be undertaken.

Results The database search retrieved 9439 references. 83 full texts were screened for inclusion and 19 papers were included in the data extraction and synthesis process.

A large US study found the prevalence of children with CCC in PICU to be 53\% (range 22.4-70.6\%). The smaller study from Greece found a prevalence of $37.8 \%$ and a multi- country study found a prevalence of $67 \%$.

The within PICU mortality rates were highest for the haematopoietic stem cell transplant (HSCT) patients (45-69\%) with the studies on oncology patients alone having mortality rates of $16-27 \%$.

Four of the studies presenting post PICU mortality data were for patients admitted to PICU post HSCT (mortality rate ranged from 67-85\%), three were on oncology patients (mortality ranged from $17-48 \%$ ) and one was children with severe neurological impairment requiring mechanical ventilation $(50 \%$ mortality one year post PICU).

Conclusions Children with CCC formed a large but variable proportion of PICU admissions in the US and had higher within PICU mortality. Children admitted to PICU post HSCT had very high within and post PICU mortality rates.

There were no studies undertaken in the UK and none using the LLC definition to define their population of interest. There were no large studies which assessed long term mortality or outcome after PICU. 